\title{
Interactions between food hazards and intestinal barrier: impact on foodborne diseases
}

\begin{abstract}
The intestine is an important digestive organ of the human body, and its barrier is the guardian of the body from the external environment. The impairment of the intestinal barrier is believed to be an important determinant in various foodborne diseases. Food hazards can lead to the occurrence of many foodborne diseases represented by inflammation. Therefore, understanding the mechanisms of the impact of the food hazards on intestinal barriers is essential for promoting human health. This review examined the relationship between food hazards and the intestinal barrier in three aspects: apoptosis, imbalance of gut microbiota, and pro-inflammatory cytokines. The mechanism of dysfunctional gut microbiota caused by food hazards was also discussed. This review discusses the interaction among food hazards, intestinal barrier, and foodborne diseases and, thus, offers a new thought to deal with foodborne disease.
\end{abstract}

Keyword: Intestinal barrier; Food hazards; Apoptosis; Gut microbiota; Pro-inflammatory cytokines; Foodborne diseases 